\title{
An epidemic model of childhood disease dynamics with maturation delay and latent period of infection
}

\author{
Harkaran Singh $^{1,2} \cdot$ Joydip Dhar $^{3} \cdot$ Harbax Singh Bhatti $^{4} \cdot$ Sumit Chandok $^{5}$
}

Received: 1 April 2016/Accepted: 12 April 2016/Published online: 28 April 2016

(C) Springer International Publishing Switzerland 2016

\begin{abstract}
In the present study, an epidemic model is proposed with maturation delay and latent period of infection, keeping in view the childhood disease dynamics and studied the asymptotic behavior of the model for all the feasible equilibrium states. The criterion for local stability of the system around steady states are established in terms of delay, latent period and system parameters. Further explored the possibility of Hopf bifurcation at the endemic equilibrium state and threshold is determined. We also performed the sensitivity analysis of the state variables at the endemic equilibrium state with respect to the model parameters and identified the respective sensitive indices. Further numerical simulations have been carried out to justify our analytic findings.
\end{abstract}

Keywords Epidemic model $\cdot$ Maturation delay $\cdot$ Latent period of infection $\cdot$ Hopf bifurcation $\cdot$ Sensitivity analysis

Harkaran Singh

harkaran78@yahoo.in

IKG-Punjab Technical University, Kapurthala 144601, India

2 Department of Applied Sciences, Khalsa College of Engineering and Technology, Amritsar 143001, Punjab, India

3 Department of Applied Sciences, ABV-Indian Institute of Information Technology and Management, Gwalior 474015, M.P., India

4 Department of Applied Sciences, B.B.S.B. Engineering College, Fatehgarh Sahib 140406, Punjab, India

5 School of Mathematics,Thapar University, Patiala 147004, Punjab, India

\section{Introduction}

Epidemics is defined as a rapid spread of a infectious disease that infects a large portion of the population in a particular region and this study of disease dynamics is a dominating theme for many biologists and mathematicians (Singh et al. 2016; Samanta 2010; Zhang et al. 2013; Xu and Ma 2010; Zhou and Cui 2011; Kang and Fu 2015; Upadhyay and Roy 2014; Bansal and Meyers 2012; Liu et al. 2013; Gao et al. 2013; Tharakaraman et al. 2013; Chao et al. 2012; Safi and Gumel 2013; Hu et al. 2012, 2014; Alexander and Moghadas 2005; Kaddar et al. 2010; Zhang et al. 2009; Samsuzzoha et al. 2013; Sahu and Dhar 2012; He et al. 2013; Sun et al. 2011; Liu et al. 2007; Tchuenche et al. 2011; Liu and Cui 2008; Cui et al. 2008a, b; Funk et al. 2009). Further, there are a number of diseases, which affects mostly the children, for example, Rubella, Measles, Chickenpox, Polio, Mumps, etc., because premature (child) population is more prone to the diseases than the mature (adult) population Reef et al. (2002). Therefore, in disease dynamics the population can be divided into two major categories: pre-mature population and mature population. The pre-mature population takes a constant time to become mature and this constant time is called maturation delay. Also, in disease dynamics, disease cannot spread instantaneously, it will take some time in the host body before the outbreak and that time period is known as the latent period of the particular disease. The simplest disease models cannot capture the rich variety of dynamics and the inclusion of temporal delays in these models makes them more realistic (Beretta and Takeuchi 1995; Brauer 1990; Jin and Ma 2006; Ma et al. 2004).

Keeping in view of the childhood disease dynamics, in this paper, we proposed and analyzed an epidemic model incorporating maturation delay and latent period of 
infection. This paper is organized as follows: in Sect. 2, formulation of epidemic model is presented. In Sect. 3, positivity and boundedness of the system has been obtained. In Sect. 4, the system is analyzed for the asymptotic stability at all the feasible equilibrium states and obtained the conditions for the existence of Hopf bifurcation at the endemic equilibrium state. In Sect. 5, the sensitivity analysis of the state variables at endemic equilibrium state with respect to model parameters is performed. In Sect. 6, we presented some numerical simulations to support our analytical findings and in the last section, a brief conclusion is given.

\section{Formulation of mathematical model}

The assumptions of the proposed model are:

(i) The population exhibit age structure and the total population at time $t$ is divided into three mutually exclusive compartments, namely, pre-mature $(P)$, mature $(M)$ and infected $(I)$.

(ii) $\quad \tau_{1}$ is maturation delay (i.e. the pre-mature period) and $d_{1}$ is the death rate of pre-mature population. The transformation from pre-mature to mature population is $\gamma e^{-d_{1} \tau_{1}} M\left(t-\tau_{1}\right)$, where $\gamma$ is the birth rate of pre-mature, who was born at time $t-\tau_{1}$ and survive at time $t$.

(iii) $\tau_{2}$ is a latent period of infection.

(iv) No vertical transmission of the disease from mature to pre-mature compartment.

(v) Treatment limitation is shown as the overcrowding in disease compartment.

(vi) Resource limitation is shown as the overcrowding in mature compartment.

The proposed system is of the form:

$\frac{d P}{d t}=\gamma M-d_{1} P-\gamma e^{-d_{1} \tau_{1}} M\left(t-\tau_{1}\right)-\alpha P\left(t-\tau_{2}\right) I$,

$\frac{d M}{d t}=\gamma e^{-d_{1} \tau_{1}} M\left(t-\tau_{1}\right)-d_{2} M-\beta_{1} M^{2}$,

$\frac{d I}{d t}=\alpha P\left(t-\tau_{2}\right) I-d_{3} I-\beta_{2} I^{2}$,

with initial conditions:

$$
\begin{gathered}
P(\theta)=\phi_{1}(\theta), M(\theta)=\phi_{2}(\theta), I(\theta)=\phi_{3}(\theta), \\
\phi_{1}(0)>0, \phi_{2}(0)>0, \phi_{3}(0)>0
\end{gathered}
$$

where $\theta \in[-\tau, 0]$ and $\phi_{1}(\theta), \phi_{2}(\theta), \phi_{3}(\theta) \in \mathcal{C}([-\tau, 0]$, $\left.R_{+}^{3}\right)$, the Banach space of continuous functions mapping the interval $[-\tau, 0]$ into $R_{+}^{3}$, where $R_{+}^{3}=\left\{\left(x_{1}, x_{2}, x_{3}\right)\right.$ : $\left.x_{i} \geq 0, i=1,2,3\right\}$.
Here $d_{2}$ and $d_{3}$ are the death rates of mature and infected populations respectively; $\alpha$ is the rate of infection to the pre-mature individuals; $\beta_{1}$ and $\beta_{2}$ are respectively the overcrowding rates of mature and infected individuals.

\section{Positivity and boundedness of the system}

We state and prove the following lemmas for the positivity and boundedness of the solution of the system (1) (2) (3):

Lemma 1 The solution of the system (1)-(3) with initial conditions are positive, for all $t \geq 0$.

Proof Let $(P(t), M(t), I(t))$ be a solution of the system (1)-(3) with initial conditions. Let us consider $I(t)$ for $t \in\left[0, \tau^{*}\right]$, where $\tau^{*}=\min \left\{\tau_{1}, \tau_{2}\right\}$. We obtain from the equation (3) that

$\frac{d I}{d t} \geq-d_{3} I-\beta_{2} I^{2}$

it follows that

$I(t) \geq \frac{d_{3} I(0)}{\beta_{2} I(0)\left(e^{d_{3} t}-1\right)+d_{3} e^{d_{3} t}}>0$.

For $t \in\left[0, \tau^{*}\right]$, the equation (2) can be rewritten as

$\frac{d M}{d t} \geq-d_{2} M-\beta_{1} M^{2}$

which implies that

$M(t) \geq \frac{d_{2} M(0)}{\beta_{1} M(0)\left(e^{d_{2} t}-1\right)+d_{2} e^{d_{2} t}}>0$.

The equation (1) for $t \in\left[0, \tau^{*}\right]$ can be rewritten as

$\frac{d P}{d t} \geq-d_{1} P-\gamma e^{-d_{1} \tau_{1}} M\left(t-\tau_{1}\right)-\alpha P\left(t-\tau_{2}\right) I$,

which evidences that

$P(t)=e^{-\tilde{I}}\left[P(0)-\int_{0}^{t} \gamma e^{-d_{1} \tau_{1}} M\left(r-\tau_{1}\right) e^{\tilde{I}} d r\right]>0$,

where $\tilde{I}=\int_{0}^{t}\left(d_{1}+\alpha I(s)\right) d s$.

In the similar way, we can treat the intervals $\left[\tau^{*}, 2 \tau^{*}\right], \ldots,\left[n \tau^{*},(n+1) \tau^{*}\right], n \in N$. Thus by induction, we establish that $P(t)>0, M(t)>0$ and $I(t)>0$ for all $t \geq 0$.

Lemma 2 The solution of the system of equations (1)-(3) with initial conditions is uniformly bounded in $\Omega$, where

$\Omega=\left\{(P, M, I): 0 \leq P(t)+M(t)+I(t) \leq \frac{k_{2}}{k_{1}}\right\}$,

$k_{1}=\min \left\{d_{1}, d_{2}, d_{3}\right\}$ and $k_{2}=\frac{\gamma^{2}}{4 \beta_{1}}$. 
Proof Let $V(t)=P(t)+M(t)+I(t)$. Calculating the derivative of $V(t)$ with respect to $t$, we have

$$
\begin{aligned}
\frac{d V(t)}{d t} & =\gamma M-d_{1} P-d_{2} M-\beta_{1} M^{2}-d_{3} I-\beta_{2} I^{2} \\
& \leq \gamma M-d_{1} P-d_{2} M-\beta_{1} M^{2}-d_{3} I .
\end{aligned}
$$

Taking $k_{1}=\min \left\{d_{1}, d_{2}, d_{3}\right\}$, we obtain that

$\frac{d V(t)}{d t}+k_{1} V \leq \gamma M-\beta_{1} M^{2}$

There exists a positive constant $k_{2}=\frac{\gamma^{2}}{4 \beta_{1}}$, such that

$\frac{d V(t)}{d t}+k_{1} V \leq k_{2}$

Thus we get

$0<V(t) \leq V(0) e^{-k_{1} t}+\frac{k_{2}}{k_{1}}$.

As $t \rightarrow \infty$, we have

$0 \leq V(t) \leq \frac{k_{2}}{k_{1}}$.

Therefore, $V(t)$ is bounded. So, each solution of the system (1)-(3) is bounded.

\section{Dynamical behavior of the system}

The system (1)-(3) has three non-negative equilibrium points:

(i) Trivial equilibrium $E_{0}(0,0,0)$ always exists.

(ii) Boundary equilibrium $E_{1}\left(P_{1}, M_{1}, 0\right)$ exists, if $\left(H_{1}\right)$ holds, where $P_{1}=\frac{\gamma\left(1-e^{-d_{1} \tau_{1}}\right)\left(\gamma e^{-d_{1} \tau_{1}}-d_{2}\right)}{\beta_{1} d_{1}}, \quad M_{1}=$ $\frac{\left(\gamma e^{-d_{1} \tau_{1}}-d_{2}\right)}{\beta_{1}}$ and

$$
\left(\mathbf{H}_{1}\right): \tau_{1}<\frac{1}{d_{1}} \ln \left(\frac{\gamma}{d_{2}}\right):=\tau_{10} .
$$

(iii) Endemic equilibrium $E^{*}\left(P^{*}, M^{*}, I^{*}\right)$ exists, if $\left(H_{1}\right), \quad\left(H_{2}\right)$ and $\left(H_{3}\right)$ holds, where $M^{*}=$ $\frac{\gamma e^{-d_{1} \tau_{1}}-d_{2}}{\beta_{1}}, I^{*}=\frac{\alpha P^{*}-d_{3}}{\beta_{2}}$ and $P^{*}$ satisfy

$$
A P^{* 2}-B P^{*}-C=0
$$

and

$$
\begin{aligned}
& A=\alpha^{2} \beta_{1}, \\
& B=\alpha \beta_{1} d_{3}-d_{1} \beta_{1} \beta_{2}, \\
& C=\beta_{2} \gamma\left(1-e^{-d_{1} \tau_{1}}\right)\left(\gamma e^{-d_{1} \tau_{1}}-d_{2}\right) .
\end{aligned}
$$

Here

$$
\left(\mathbf{H}_{2}\right): B^{2}+4 A C \geq 0, \quad\left(\mathbf{H}_{3}\right): P^{*}>\frac{d_{3}}{\alpha} .
$$

Now, if $\left(H_{1}\right)$ holds, then $M^{*}, P^{*}$ are positive and $P^{*}$ is uniquely defined by $P^{*}=\frac{B+\sqrt{B^{2}+4 A C}}{2 A}$. Also $P^{*}$ is real if $\left(H_{2}\right)$ holds. Further $I^{*}$ is positive if $\left(\mathrm{H}_{3}\right)$ holds.

Now, the transcendental polynomial equation of the second degree

$$
\lambda^{2}+p \lambda+r+(s \lambda+q) e^{-\lambda \tau}=0
$$

has been studied by Ruan (2001) and the following results have been discussed:

(A1) $\quad p+s>0$

(A2) $q+r>0$

(A3) either $s^{2}-p^{2}+2 r<0$ and $r^{2}-q^{2}>0$ or $\left(s^{2}-\right.$ $\left.p^{2}+2 r\right)^{2}<4\left(r^{2}-q^{2}\right)$;

(A4) either $r^{2}-q^{2}<0$ or $s^{2}-p^{2}+2 r>0$ and $\left(s^{2}-p^{2}\right.$ $+2 r)^{2}=4\left(r^{2}-q^{2}\right)$; either $r^{2}-q^{2}>0, s^{2}-p^{2}+2 r>0$ and $\left(s^{2}-p^{2}\right.$ $+2 r)^{2}>4\left(r^{2}-q^{2}\right)$.

Lemma 3 (see Ruan (2001)) For equation (4);

(i) If (A1)-(A3) holds, then all the roots of (4) have negative real parts for all $\tau \geq 0$.

(ii) If (A1), (A2) and (A4) hold and $\tau=\tau_{j}^{+}$, then equation (4) has a pair of purely imaginary roots $\pm i w_{+}$. When $\tau=\tau_{j}^{+}$then all roots of (4) except $\pm i w_{+}$have negative real parts.

(iii) If (A1), (A2) and (A5) hold and $\tau=\tau_{j}^{+}\left(\tau=\tau_{j}^{-}\right.$ respectively) then equation (4) has a pair of purely imaginary roots $\pm i w_{+}\left( \pm i w_{-}\right.$, respectively). Furthermore $\tau=\tau_{j}^{+}\left(\tau_{j}^{-}\right.$, respectively), then all roots of (4) except $\pm i w_{+}\left( \pm i w_{-}\right.$, respectively)have negative real parts.

Now, we will discuss the local behavior of all the equilibrium points of the system (1)-(3).

Theorem 1 The local behavior of different equilibria of the system (1)-(3) is as follows;

(i) If $\tau_{1} \geq \tau_{10}$, then trivial equilibrium $E_{0}$ is locally asymptotically stable for all $\tau_{2}$ and if $\tau_{1}<\tau_{10}$, then it is unstable.

(ii) If $\left(H_{1}\right)$ and $\left(H_{4}\right)$ holds, then boundary equilibrium $E_{1}$ is locally asymptotically stable for all $\tau_{2}$, otherwise it is unstable. 
(iii) If $\left(H_{1}\right)-\left(H_{3}\right)$ and $\left(H_{5}\right)$ holds, then the endemic equilibrium $E^{*}$ is locally asymptotically stable for all $\tau_{2}$, otherwise it is unstable.

Proof

(i) The characteristic equation for $E_{0}(0,0,0)$ is $\left(\lambda+d_{1}\right)\left(\lambda-\gamma e^{-d_{1} \tau_{1}} e^{-\lambda \tau_{1}}+d_{2}\right)\left(\lambda+d_{3}\right)=0$.

Clearly $\lambda=-d_{1}, \lambda=-d_{3}$ are negative eigenvalues. If $\gamma e^{-d_{1} \tau_{1}} \leq d_{2}$, then all the eigenvalues of (5) have negative real part. Therefore, the equilibrium $E_{0}(0,0,0)$ is locally asymptotically stable, if $\gamma e^{-d_{1} \tau_{1}} \leq d_{2}$, that is, if

$\tau_{1} \geq \frac{1}{d_{1}} \ln \left(\frac{\gamma}{d_{2}}\right)=\tau_{10}$.

Thus, if $\tau_{1} \geq \tau_{10}$, then the equilibrium $E_{0}(0,0,0)$ is locally asymptotically stable for all $\tau_{2}$. Moreover, $E_{0}(0,0,0)$ is unstable for all $\tau_{1}<\tau_{10}$, because one of the eigenvalues of (5) has positive real part.

(ii) The characteristic equation for $E_{1}\left(P_{1}, M_{1}, 0\right)$ is

$\left(\lambda+d_{1}\right) F_{1}(\lambda) F_{2}(\lambda)=0$,

where $F_{1}(\lambda)=\lambda-\alpha P_{1}+d_{3}, F_{2}(\lambda)=\lambda-\gamma e^{-d_{1} \tau_{1}}$ $e^{-\lambda \tau_{1}}+d_{2}+2 \beta_{1} M_{1}$. Now $d_{2}+2 \beta_{1} M_{1}-\gamma e^{-d_{1} \tau_{1}}$ $e^{-\lambda \tau_{1}}>0$, if $\left(H_{1}\right)$ holds. Further $d_{3}-\alpha P_{1}>0$, if $\left(H_{4}\right)$ holds, where

$\left(\mathbf{H}_{4}\right): \alpha P_{1}<d_{3}$.

Thus, if $\left(H_{1}\right)$ and $\left(H_{4}\right)$ holds, than all the eigen values of (6) have negative real parts and hence non-negative equilibrium $E_{1}\left(P_{1}, M_{1}, 0\right)$ is locally asymptotically stable for all $\tau_{2}$, otherwise it is unstable.

(iii) The characteristic equation of the Jacobian matrix at the equilibrium point $E^{*}\left(P^{*}, M^{*}, I^{*}\right)$ can be written as:

$(\lambda+A) F(\lambda)=0$,

where

$F(\lambda)=\left(\lambda+\beta_{2} I^{*}\right)\left(\lambda+d_{1}+\alpha e^{-\lambda \tau_{2}} I^{*}\right)+\alpha^{2} I^{*} P^{*} e^{-\lambda \tau_{2}}$

and

$A=\gamma e^{-d_{1} \tau_{1}}\left(2-e^{-\lambda \tau_{1}}\right)-d_{2}$

Here $A>0$ if $\left(H_{1}\right)$ holds. From (7), we obtain that $\lambda=-A$ or $F(\lambda)=0$. If $F(\lambda)=0$, then we have

$\left(\lambda+\beta_{2} I^{*}\right)\left(\lambda+d_{1}+\alpha e^{-\lambda \tau_{2}} I^{*}\right)+\alpha^{2} I^{*} P^{*} e^{-\lambda \tau_{2}}=0$.
Separating real and imaginary parts of (8) after substituting $\lambda=\xi+i \omega$, we obtain

$G_{1} G_{2}-\omega H=-\alpha^{2} I^{*} P^{*} e^{-\xi \tau_{2}} \cos \omega \tau_{2}$,

$G_{1} H+\omega G_{2}=\alpha^{2} I^{*} P^{*} e^{-\xi \tau_{2}} \sin \omega \tau_{2}$,

where

$G_{1}=\xi+\beta_{2} I^{*}$,

$G_{2}=\xi+d_{1}+\alpha I^{*} e^{-\xi \tau_{2}} \cos \omega \tau_{2}$,

$H=\omega-\alpha I^{*} e^{-\xi \tau_{2}} \sin \omega \tau_{2}$.

Thus we have

$$
\begin{aligned}
& \left(G_{1} G_{2}\right)^{2}+(\omega H)^{2}+\left(G_{1} H\right)^{2} \\
& \quad+\left(\omega G_{2}\right)^{2}=\left(\alpha^{2} I^{*} P^{*}\right)^{2} e^{-2 \xi \tau_{2}} .
\end{aligned}
$$

Now we assume that $\left(\mathbf{H}_{5}\right): \beta_{2} d_{1}>\alpha^{2} P^{*}$. We have to show that $\xi$ is negative. If possible, suppose that $\xi \geq 0$. We have

$$
\begin{aligned}
G_{1} & =\xi+\beta_{2} I^{*}, G_{2}=\xi+d_{1} \\
& +\alpha I^{*} e^{-\xi \tau_{2}} \cos \omega \tau_{2}>\xi+d_{1} .
\end{aligned}
$$

Therefore

$\left(G_{1} G_{2}\right)^{2}>\left(\alpha^{2} I^{*} P^{*}\right)^{2} e^{-2 \xi \tau_{2}}$,

which is a contradiction of (9). Therefore, $\xi<0$ if $\left(H_{5}\right)$ holds. Thus, each solution of (8) has negative real part, if $\left(H_{5}\right)$ holds. Therefore, the endemic equilibrium $E^{*}\left(P^{*}, M^{*}, I^{*}\right)$ is locally asymptotically stable if $\left(H_{1}\right)-\left(H_{3}\right)$ and $\left(H_{5}\right)$ holds for all $\tau_{2}$.

Next, we will discuss the behavior of (1)-(3) taken all possible cases of $\tau_{1}$ and $\tau_{2}$. From (8), we have

$\lambda^{2}+A_{1} \lambda+A_{2}+\left(B_{1} \lambda+B_{2}\right) e^{-\lambda \tau_{2}}+C e^{-\lambda \tau_{1}}=0$,

where

$$
\begin{aligned}
& A_{1}=d_{1}+\beta_{2} I^{*}, \\
& A_{2}=\beta_{2} d_{1} I^{*}, \\
& B_{1}=\alpha I^{*}, \\
& B_{2}=\alpha \beta_{2} I^{* 2}+\alpha^{2} I^{*} P^{*}, \\
& C=0 .
\end{aligned}
$$

Case I: If $\tau_{2}=0$, then we get

$$
\lambda^{2}+\left(A_{1}+B_{1}\right) \lambda+\left(A_{2}+B_{2}\right)=0 .
$$

In this case, all the roots of (11) have negative real parts and therefore, the endemic equilibrium $E^{*}\left(P^{*}, M^{*}, I^{*}\right)$ is locally asymptotically stable.

Case II: If $\tau_{2}>0$, then we get

$$
\lambda^{2}+p \lambda+r+(s \lambda+q) e^{-\lambda \tau_{2}}=0,
$$


where

$$
\begin{aligned}
& p=d_{1}+\beta_{2} I^{*}, \\
& r=\beta_{2} d_{1} I^{*}, \\
& s=\alpha I^{*}, \\
& q=\alpha \beta_{2} I^{* 2}+\alpha^{2} I^{*} P^{*} .
\end{aligned}
$$

We have

$$
\begin{aligned}
& q+r=\alpha \beta_{2} I^{* 2}+\alpha^{2} I^{*} P^{*}+\beta_{2} d_{1} I^{*}>0, \\
& p+s=d_{1}+\beta_{2} I^{*}+\alpha I^{*}>0, \\
& s^{2}-p^{2}+2 r=-d_{1}^{2}-\left(\beta_{2}^{2}-\alpha^{2}\right) I^{* 2}, \\
& r^{2}-q^{2}=\left(\beta_{2} d_{1} I^{*}\right)^{2}-\left(\alpha \beta_{2} I^{* 2}+\alpha^{2} I^{*} P^{*}\right)^{2} .
\end{aligned}
$$

We have $s^{2}-p^{2}+2 r<0$, if $\left(H_{6}\right)$ holds and $r^{2}-q^{2}>0$, if $\left(H_{7}\right)$ holds, where

$$
\begin{aligned}
& \left(\mathbf{H}_{\mathbf{6}}\right):\left(\alpha^{2}-\beta_{2}^{2}\right) I^{* 2}<d_{1}^{2}, \\
& \left(\mathbf{H}_{7}\right): \frac{d_{3}}{\alpha}<P^{*}<\frac{1}{2 \alpha}\left(d_{3}+\frac{\beta_{2} d_{1}}{\alpha}\right) .
\end{aligned}
$$

Using Lemma 3, if $\left(H_{6}\right)$ and $\left(H_{7}\right)$ holds, then all the roots of (1)-(3) have negative real parts for all $\tau_{2}$ and hence $E^{*}\left(P^{*}, M^{*}, I^{*}\right)$ is locally asymptotically stable.

Further $r^{2}-q^{2}<0$, if $\left(\mathbf{H}_{8}\right): P^{*}>\frac{1}{2 \alpha}\left(d_{3}+\frac{\beta_{2} d_{1}}{\alpha}\right)$ holds.

Using Lemma 3, if $\left(H_{8}\right)$ holds, then the system (1)-(3) has a pair of purely imaginary roots.

Put $\lambda=i w$ in (12), we get

$$
(i w)^{2}+p(i w)+r+(i w s+q) e^{-i w \tau_{2}}=0 .
$$

Equating real and imaginary parts, we get

$-w^{2}+r+s w \sin w \tau_{2}+q \cos w \tau_{2}=0$,

$p w+s w \cos w \tau_{2}-q \sin w \tau_{2}=0$.

Solving (13) and (14), we get

$$
\begin{aligned}
& \sin w \tau_{2}=\frac{s w^{3}+(p q-r s) w}{s^{2} w^{2}+q^{2}}, \\
& \cos w \tau_{2}=\frac{(q-p s) w^{2}-q r}{s^{2} w^{2}+q^{2}}
\end{aligned}
$$

and

$$
w^{4}+\left(p^{2}-2 r-s^{2}\right) w^{2}+\left(r^{2}-q^{2}\right)=0 .
$$

We define

$$
F(w)=w^{4}+\left(p^{2}-2 r-s^{2}\right) w^{2}+\left(r^{2}-q^{2}\right)=0 .
$$

Now, $F(0)=\left(r^{2}-q^{2}\right)<0$, if $\left(H_{8}\right)$ holds. By Descartes' rule of sign, there is at least one positive root of $F(w)=0$. Let $w_{0}$ is the positive root of $F(w)=0$.
From (16), we get

$$
\tau_{2 k}^{+}=\frac{1}{w_{0}}\left[\cos ^{-1}\left(\frac{(q-p s) w_{0}^{2}-q r}{s^{2} w_{0}^{2}+q^{2}}\right)+2 k \pi\right]
$$

where $k=0,1,2, \ldots$.

Now, differentiating (12) with respect to $\tau_{2}$, we get

$$
\frac{d \lambda}{d \tau_{2}}=\frac{\lambda(s \lambda+q) e^{-\lambda \tau_{2}}}{2 \lambda+p-(s \lambda+q) \tau_{2} e^{-\lambda \tau_{2}}+s e^{-\lambda \tau_{2}}} .
$$

At $\lambda=i w_{0}$ and $\tau_{2}=\tau_{20}^{+}$, we have

$$
\begin{aligned}
& \operatorname{Re}\left(\frac{d \lambda}{d \tau_{2}}\right)^{-1} \\
& \quad=\frac{\left(s w_{0} p+2 w_{0} q\right) \cos w_{0} \tau_{2}+\left(p q-2 s w_{0}^{2}\right) \sin w_{0} \tau_{2}+s^{2} w_{0}}{w_{0}\left(q^{2}+s^{2} w_{0}^{2}\right)} .
\end{aligned}
$$

Simplifying (18), we have

$\operatorname{Re}\left[\left(\frac{d \lambda}{d \tau_{2}}\right)^{-1}\right]_{\tau_{2}=\tau_{20}^{+}} \neq 0$,

for $p q>2 s w_{0}^{2}$, which is one of the sufficient condition.

Theorem 2 Let $\left(H_{1}\right)-\left(H_{3}\right)$ holds. For the system (1)-(3), we have

(i) If $\left(H_{6}\right)$ and $\left(H_{7}\right)$ holds, then the endemic equilibrium $E^{*}\left(P^{*}, M^{*}, I^{*}\right)$ is locally asymptotically stable for all $\tau_{2}$.

(ii) If $\left(H_{8}\right)$ holds, then the endemic equilibrium $E^{*}\left(P^{*}, M^{*}, I^{*}\right)$ is locally asymptotically stable for all $\tau_{2} \in\left[0, \tau_{20}^{+}\right)$, and unstable when $\tau_{2} \geq \tau_{20}^{+}$.

\section{Sensitivity analysis}

In this section, we perform the sensitivity analysis of the endemic equilibrium with respect to model parameters, for a particular set of parameters $\gamma=0.8, \alpha=0.09$, $\beta_{1}=0.2, \beta_{2}=0.3, d_{1}=0.05, d_{2}=0.01, d_{3}=0.01, \tau_{1}=$ 12.2 and $\tau_{2}=7.23$. The normalized sensitive indices of the endemic equilibrium with respect to parameters are shown in Table 1. From the Table 1, it is observed that $\gamma$, $\beta_{2}, d_{3}$ have a positive impact on the $P^{*}$ and the rest of the parameters have a negative impact. Moreover $\gamma$ and $\alpha$ are most sensitive parameter to $P^{*}$, hence, the significant change in $P^{*}$ is observed by small changes in these parameters. Again $\gamma$ and $\beta_{1}$ are most sensitive parameters to both $M^{*}$ and $I^{*}$. 
Table 1 The sensitivity indices $\gamma_{y_{j}}^{x_{i}}=\frac{\partial x_{i}}{\partial y_{j}} \times \frac{y_{j}}{x_{i}}$ of the state variables of the system (1)-(3) to the parameters $y_{j}$ for the parameter values $\gamma=$ $0.8, \alpha=0.09, \quad \beta_{1}=0.2, \beta_{2}=0.3, d_{1}=0.05, d_{2}=0.01, d_{3}=0.01$, $\tau_{1}=12.2, \tau_{2}=7.2$

\begin{tabular}{llll}
\hline Parameter $\left(y_{j}\right)$ & $\gamma_{y_{j}}^{P^{*}}$ & $\gamma_{y_{j}}^{M^{*}}$ & $\gamma_{y_{j}}^{I^{*}}$ \\
\hline$\gamma$ & 1.17394 & 1.02355 & 1.20326 \\
$\alpha$ & -0.829488 & 0 & 0.17477 \\
$\beta_{1}$ & -0.58014 & -1 & -1.59463 \\
$\beta_{2}$ & 0.409629 & 0 & 0.41986 \\
$d_{1}$ & -0.111654 & -0.624364 & -0.114443 \\
$d_{2}$ & -0.0136606 & -0.0235471 & -0.0140018 \\
$d_{3}$ & 0.0102307 & 0 & -0.0144893 \\
\hline
\end{tabular}

\section{Numerical simulations}

We perform the numerical simulations of the system (1)(3) keeping the parameters $\gamma=0.8, \alpha=0.04, \beta_{1}=0.8$, $\beta_{2}=0.3$ as fixed and varying the parameters $d_{1}, d_{2}$ and $d_{3}$. We use initial population sizes as $P_{0}=0.3, M_{0}=0.9$ and $I_{0}=0.4$.

The trivial equilibrium $E_{0}(0,0,0)$ is locally asymptotically stable for the parameter values $d_{1}=0.3, d_{2}=0.01$ and $d_{3}=0.09$, when $\tau_{1}=15 \geq \bar{\tau}_{10}=14.6$ and $\tau_{2}=7.2$ (see Fig. 1). For the same set of parametric values the boundary equilibrium $E_{1}\left(P_{1}, M_{1}, 0\right)$ is locally asymptotically stable when $\tau_{1}=12.2<\bar{\tau}_{10}=14.6$ and $\tau_{2}=7.2$ as shown in Fig. 2. Further, the endemic equilibrium

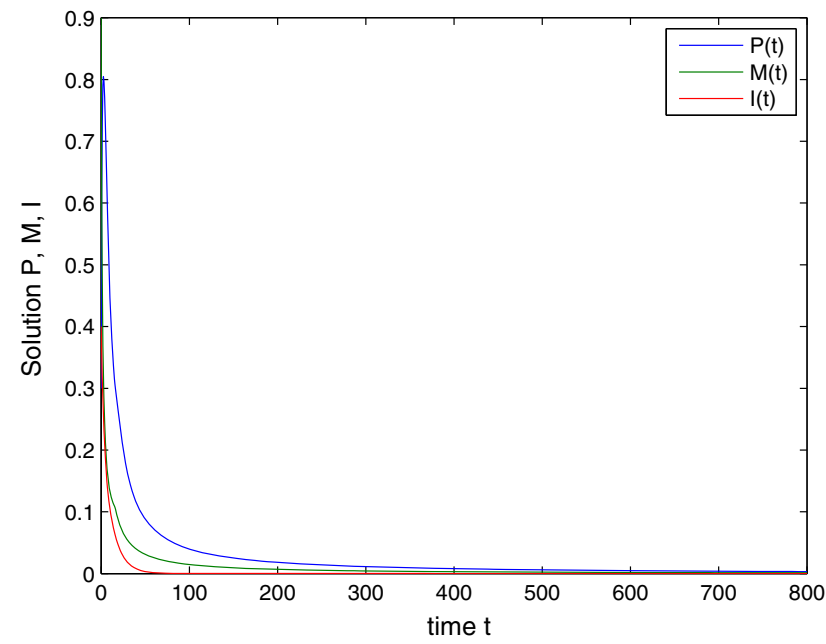

Fig. $1 E_{0}$ is stable for parameter values $\gamma=0.8, \alpha=0.04, \beta_{1}=$ $0.8, \beta_{2}=0.3, d_{1}=0.3, d_{2}=0.01, d_{3}=0.09, \tau_{1}=15>\bar{\tau}_{10}=14.6$ and $\tau_{2}=7.2$

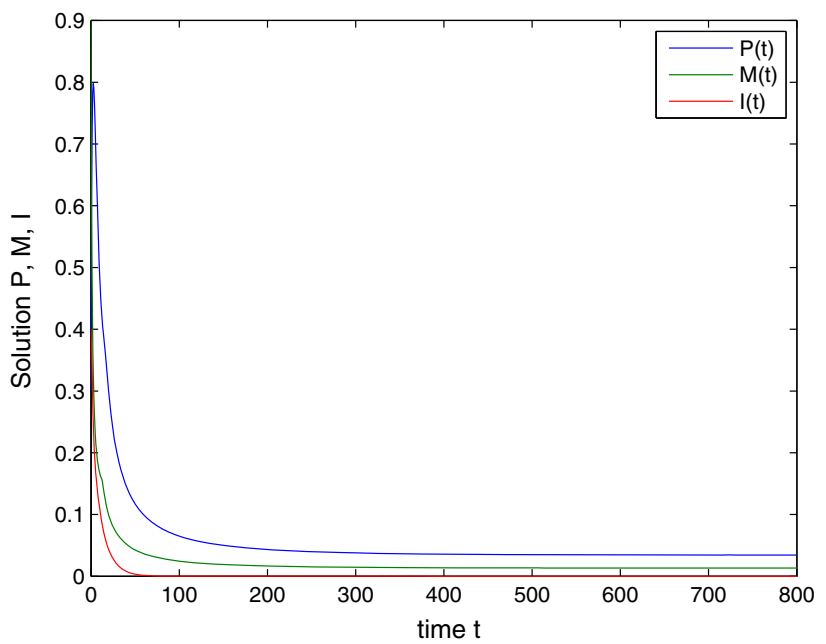

Fig. $2 E_{1}$ is stable for parameter values $\gamma=0.8, \alpha=0.04, \beta_{1}=$ $0.8, \beta_{2}=0.3, d_{1}=0.3, d_{2}=0.01, d_{3}=0.09, \tau_{1}=12.2<\bar{\tau}_{10}=14.6$ and $\tau_{2}=7.2$

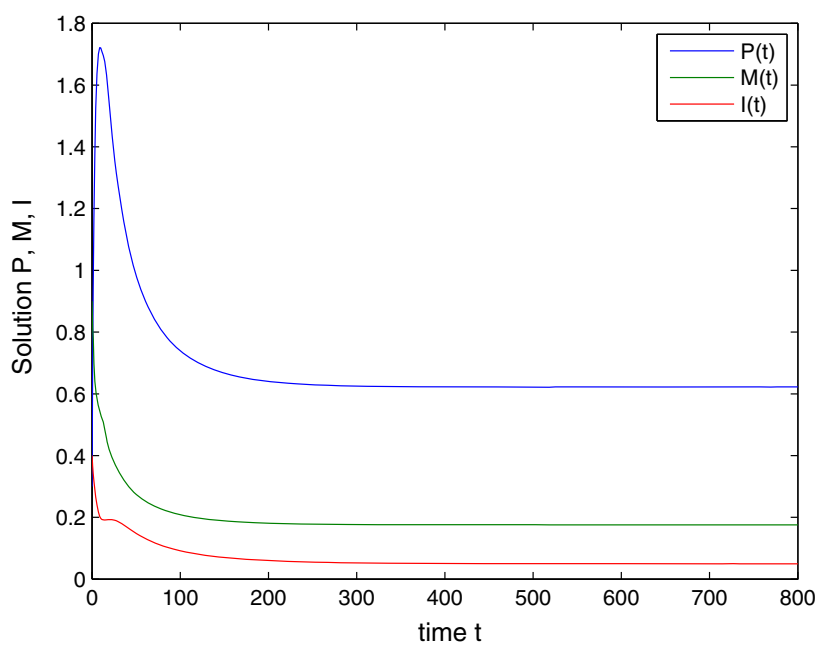

Fig. $3 E^{*}$ is stable for parameter values $\gamma=0.8, \alpha=0.04, \beta_{1}=0.2$, $\beta_{2}=0.3, d_{1}=0.2, d_{2}=0.05, d_{3}=0.01, \tau_{1}=11.2<\bar{\tau}_{10}=13.86$ and $\tau_{2}=7.2$

$E^{*}\left(P^{*}, M^{*}, I^{*}\right)$ is locally asymptotically stable when $\tau_{1}=$ $11.2<\bar{\tau}_{10}=13.86$ and $\tau_{2}=7.2$ for parameter values $d_{1}=0.2, d_{2}=0.05$ and $d_{3}=0.01$ (see Fig. 3). These results show that the Theorem 1 is true.

Again, we take parameter values as $d_{1}=0.05$, $d_{2}=0.01, d_{3}=0.01$ and $\tau_{1}=12.2$. The endemic equilibrium $E^{*}\left(P^{*}, M^{*}, I^{*}\right)$ is stable when $\tau_{2}=7.2<\tau_{20}^{+}=7.25$ as shown in Fig. 4. Further, $E^{*}\left(P^{*}, M^{*}, I^{*}\right)$ is unstable and Hopf bifurcation appears when $\tau_{2}=7.35 \geq \tau_{20}^{+}=7.25$ as shown in Fig. 5, which is in accordance with the results stated in Theorem 2. 

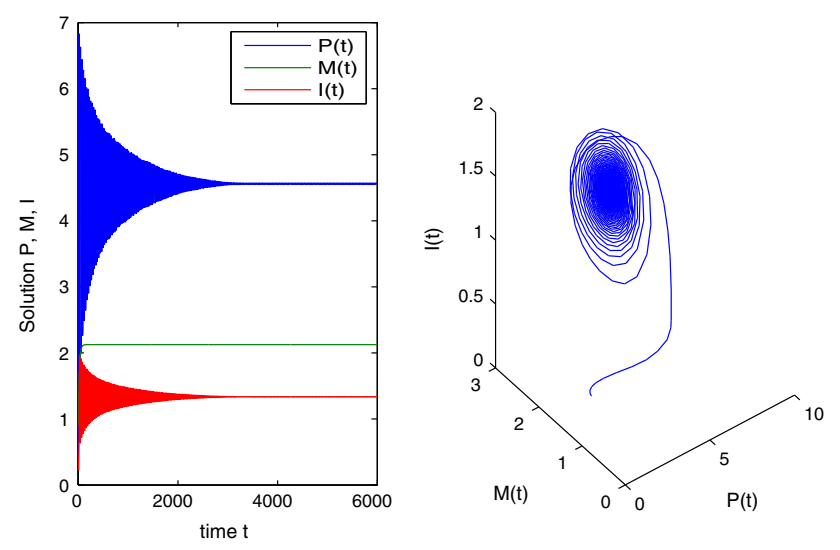

Fig. $4 E^{*}$ is stable for parameter values $\gamma=0.8, \alpha=0.09, \beta_{1}=0.2$, $\beta_{2}=0.3, d_{1}=0.05, d_{2}=0.01, d_{3}=0.01, \tau_{1}=12.2, \tau_{2}=7.2<\tau_{20}^{+}$ $=7.25$
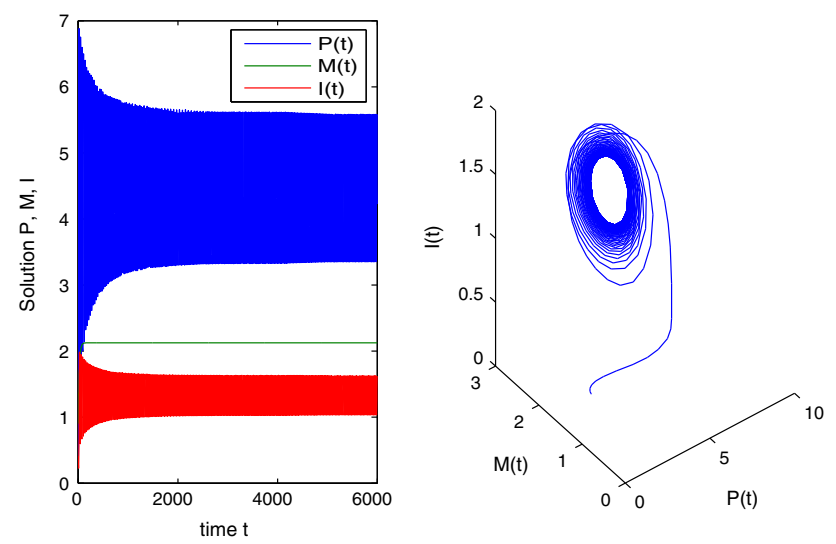

Fig. $5 E^{*}$ is unstable for parameter values $\gamma=0.8, \alpha=0.09, \beta_{1}=$ $0.2, \beta_{2}=0.3, d_{1}=0.05, d_{2}=0.01, d_{3}=0.01, \tau_{1}=12.2, \tau_{2}=$ $7.35>\tau_{20}^{+}=7.25$

\section{Conclusions}

In this paper, an epidemic model for childhood disease with maturation delay and latent period of infection is proposed. The asymptotic stability of the model is investigated at all the feasible equilibrium states. The existence of Hopf bifurcation at the endemic equilibrium state is explored. The endemic equilibrium is locally asymptotically stable for all $\tau_{2} \in\left[0, \tau_{20}^{+}\right)$, and exhibits Hopf bifurcation, when the latent period $\left(\tau_{2}\right)$ is greater than or equal to some critical value $\left(\tau_{20}^{+}\right)$under specific conditions. Finally, the normalized forward sensitivity indices are calculated for state variables at endemic equilibrium with respect to various parameters. Numerical simulations of the system are performed with a particular set of parameters to justify our analytic findings.

\section{References}

Alexander ME, Moghadas SM (2005) Bifurcation analysis of an sirs epidemic model with generalized incidence. SIAM J Appl Math 65(5):1794-1816

Bansal S, Meyers LA (2012) The impact of past epidemics on future disease dynamics. J Theor Biol 309:176-184

Beretta E, Takeuchi Y (1995) Global stability of an sir epidemic model with time delays. J Math Biol 33(3):250-260

Brauer F (1990) Models for the spread of universally fatal diseases. J Math Biol 28(4):451-462

Chao DL, Bloom JD, Kochin BF, Antia R, Longini IM (2012) The global spread of drug-resistant influenza. J R Soc Interface 9(69):648-656

Cui J, Sun Y, Zhu H (2008a) The impact of media on the control of infectious diseases. J Dyn Differ Equ 20(1):31-53

Cui J-a, Tao X, Zhu H (2008b) An sis infection model incorporating media coverage. Rocky Mt J Math 38:1323-1334. doi:10.1216/ RMJ-2008-38-5-1323

Funk S, Gilad E, Watkins C, Jansen VA (2009) The spread of awareness and its impact on epidemic outbreaks. Proc Natl AcadSci 106(16):6872-6877

Gao R, Cao B, Hu Y, Feng Z, Wang D, Hu W, Chen J, Jie Z, Qiu H, $\mathrm{Xu} \mathrm{K}$ et al (2013) Human infection with a novel avian-origin influenza a (h7n9) virus. New Eng J Med 368(20):1888-1897

He Y, Gao S, Xie D (2013) An sir epidemic model with time-varying pulse control schemes and saturated infectious force. Appl Math Model 37(16):8131-8140

Hu Z, Teng Z, Jiang H (2012) Stability analysis in a class of discrete sirs epidemic models. Nonlinear Analy Real World Appl 13(5):2017-2033

Hu Z, Teng Z, Zhang L (2014) Stability and bifurcation analysis in a discrete sir epidemic model. Math Comput Simul 97:80-93

Jin Z, Ma Z (2006) The stability of an sir epidemic model with time delays. Math Biosci Eng MBE 3(1):101-109

Kaddar A, Abta A, Alaoui H (2010) Stability analysis in a delayed SIR epidemic model with a saturated incidence rate. Nonlinear Anal Model Control 15(3):299-306

Kang H, Fu X (2015) Epidemic spreading and global stability of an SIS model with an infective vector on complex networks. Commun Nonlinear Sci Numer Simul 27(1):30-39

Liu R, Wu J, Zhu H (2007) Media/psychological impact on multiple outbreaks of emerging infectious diseases. Comput Math Methods Med 8(3):153-164

Liu X, Liu Y, Zhang Y, Chen Z, Tang Z, Xu Q, Wang Y, Zhao P, Qi Z (2013) Pre-existing immunity with high neutralizing activity to 2009 pandemic h1n1 influenza virus in shanghai population. PloS One 8(3):e58810

Liu Y, Cui J-A (2008) The impact of media coverage on the dynamics of infectious disease. Int J Biomath 1(01):65-74

Ma W, Song M, Takeuchi Y (2004) Global stability of an sir epidemic model with time delay. Appl Math Lett 17(10):1141-1145

Reef SE, Frey TK, Theall K, Abernathy E, Burnett CL, Icenogle J, McCauley MM, Wharton M (2002) The changing epidemiology of rubella in the 1990s: on the verge of elimination and new challenges for control and prevention. Jama 287(4):464-472

Ruan S (2001) Absolute stability, conditional stability and bifurcation in kolmogorov-type predator-prey systems with discrete delays. Q Appl Math 59(1):159-174

Safi MA, Gumel AB (2013) Dynamics of a model with quarantineadjusted incidence and quarantine of susceptible individuals. J Math Anal Appl 399(2):565-575

Sahu GP, Dhar J (2012) Analysis of an sveis epidemic model with partial temporary immunity and saturation incidence rate. Appl Math Model 36(3):908-923 
Samanta G (2010) Analysis of a nonautonomous hiv/aids epidemic model with distributed time delay. Math Model Anal 15(3):327-347

Samsuzzoha M, Singh M, Lucy D (2013) Uncertainty and sensitivity analysis of the basic reproduction number of a vaccinated epidemic model of influenza. Appl Math Model 37(3):903-915

Singh H, Dhar J, Bhatti HS (2016) Dynamics of a prey-generalized predator system with disease in prey and gestation delay for predator. Model Earth Syst Environ 2(2):1-9

Sun C, Yang W, Arino J, Khan K (2011) Effect of media-induced social distancing on disease transmission in a two patch setting. Math Biosci 230(2):87-95

Tchuenche JM, Dube N, Bhunu CP, Bauch CT et al (2011) The impact of media coverage on the transmission dynamics of human influenza. BMC Public Health 11(Suppl 1):S5

Tharakaraman K, Jayaraman A, Raman R, Viswanathan K, Stebbins NW, Johnson D, Shriver Z, Sasisekharan V, Sasisekharan R
(2013) Glycan receptor binding of the influenza a virus $\mathrm{h} 7 \mathrm{n} 9$ hemagglutinin. Cell 153(7):1486-1493

Upadhyay RK, Roy P (2014) Spread of a disease and its effect on population dynamics in an eco-epidemiological system. Commun Nonlinear Sci Numer Simul 19(12):4170-4184

Xu R, Ma Z (2010) Global stability of a delayed seirs epidemic model with saturation incidence rate. Nonlinear Dyn 61(1-2):229-239

Zhang T, Liu J, Teng Z (2009) Bifurcation analysis of a delayed sis epidemic model with stage structure. Chaos Solitons Fractals 40(2):563-576

Zhang T, Meng X, Song Y, Zhang T (2013) A stage-structured predator-prey si model with disease in the prey and impulsive effects. Math Model Anal 18(4):505-528

Zhou X, Cui J (2011) Analysis of stability and bifurcation for an seiv epidemic model with vaccination and nonlinear incidence rate. Nonlinear Dyn 63(4):639-653 